\title{
The Distribution of Ideal Class Numbers of Real Quadratic Fields
}

\author{
By M. D. Hendy
}

\begin{abstract}
A table of class numbers of real quadratic number fields $Q(\sqrt{ } d)$ with squarefree determinant $d, 1000<d<100000$ is examined and several analyses of the distribution of the class numbers, and the number of classes per genus are made. From these, two conjectures on the possible distribution of the class numbers as $d \rightarrow \infty$ are made, which are consistent with Gauss's related conjecture.
\end{abstract}

1. Introduction. In $1965 \mathrm{~K}$. E. Kloss [4] announced the production of a table listing the primes $p \equiv 1(\bmod 4), p<120000$ which are the determinants of real quadratic number fields whose domains of integers are unique factorization domains. He notes that approximately $80 \%$ of the primes considered are in the table. In addition, a table of ideal class numbers $h(p)$ for $Q(\sqrt{ } p), p \equiv 1(\bmod 4), p$ prime $<95000$ was produced.

Subsequently, a table of class numbers [5] containing $h(p)$ for the first 5000 primes $p \equiv 1(\bmod 4),(5 \leqslant p \leqslant 105269)$ was deposited in the UMT file. In his review of this file, D. Shanks [7] analyzed the data in two ways. Firstly, he followed up Kloss's observation on the proportion of fields with $h(p)=1$, producing a table showing the number of primes $p$ in each portion of 1000 values, which had a given class number. He noted that the proportion of values $p$ with class numbers $h=1,3,5,7$ and 9 , respectively, was $80 \%, 10 \%, 3.6 \%, 2 \%$ and $1.2 \%$; and further that these proportions were remarkably stable in the smaller intervals. This distribution reinforces Gauss's related conjecture [1, Section 304] that the number of fields with one class per genus is a fixed proportion of the population as the determinant goes to infinity. Shanks raises the question on what is the nature of this distribution.

Another more recent tabulation, by Richard B. Lakein [6] , is a table of class numbers of the quartic fields $K=F\left(\pi^{1 / 2}\right)$ where $F=Q(i)$ for 5000 Gaussian primes $\pi \equiv \pm 1(\bmod 4)$. This distribution of class numbers of these fields is strikingly similar to that noted earlier by Shanks [7].

Shanks' second analysis was a table of the exceptionally large class numbers, or more specifically, those primes $p$, for which $h(p)$ is larger than any preceding class number. This list contained 12 primes ranging from $p=229(h=3)$ to $p=90001$ $(h=87)$. We can note that these values are bounded above by $\sqrt{ } p$, with the ratio $h / \sqrt{ } p$ ranging from 0.20 to 0.36 .

Received September 29, 1974; revised February 24, 1975. AMS (MOS) subject classifications (1970). Primary 12A25, $12 \mathrm{~A} 50$.

Key words and phrases. Ideal classes, continued-fraction expansion, Lagrange algorithm, ideal genera, Dirichlet series, fundamental unit. 


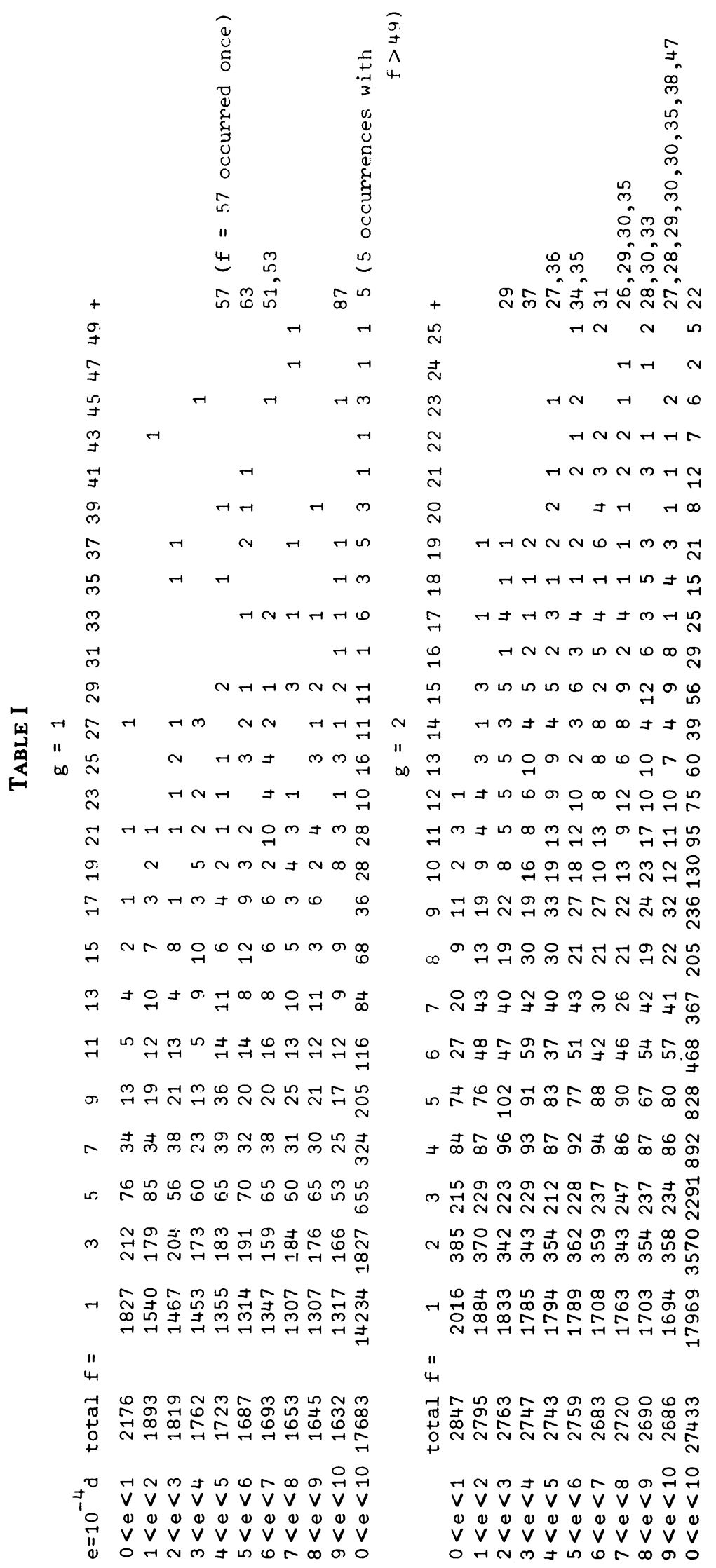




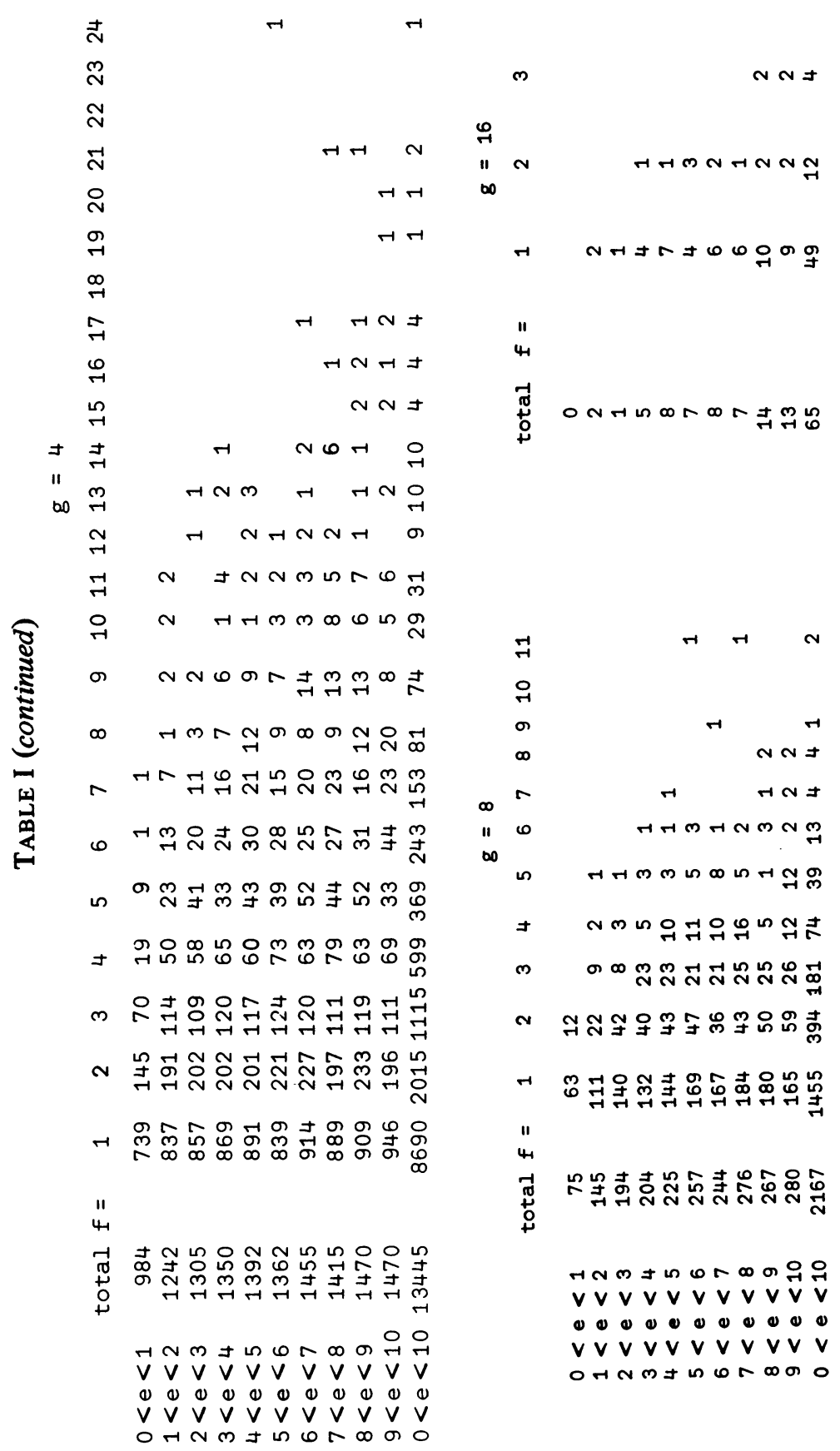


Recently the opportunity arose during the testing and early operation of a Burroughs B6700 computer to run a job which was "computer bound" for long intervals with little input output and a small usage of core.

A program was written based on a class number algorithm which is similar to Ince's procedure of counting periods. Briefly, this algorithm was based on an adaption of Lagrange's algorithm for calculating the continued fraction coefficients of a quadratic surd as described in [2]. If we apply this algorithm to the quadratic surd $\beta / b$, where $\beta$ is a primitive algebraic integer of the real quadratic field of discriminant $D$, and $b \| N(\beta)$, $b<1 / 2 D^{1 / 2}$, then we find that the sequence $Q_{n}$ in the algorithm includes the norms of all the primitive ideals in the same class as $B=(b, \beta)$, with norm $<1 / 2 D^{1 / 2}$. Hence, by using the Minkowski bound, we can calculate the class number. The program calculated the class number, $h(d)$, the number of ideal genera, $g(d)$ and the number of ideal classes of the principal genus, $f(d)$ for each field $Q(\sqrt{ } d)$ with squarefree determinant $d, 1000$ $<d<100000$. A table was produced, in 99 sections, each section giving $d, f(d), g(d)$, $h(d)$ for each squarefree $d$ in an interval of 1000 integers, followed by a count of the number fields with each combination $(g(d), f(d))$ that occurred.

2. Checking the Table. Several partial checks were made. The most important was that $g(d), h(d)$ were calculated independently, and a check was made to ensure that their ratio $f=h / g$ was integral. This check is, of course, of no value for $g=1$. Secondly, the values of $h(d)$ for the first 1227 values $1<d<2025$ were computed and compared with the corresponding class numbers in Ince's table [3]. Finally, the 16 extreme values of $h(p)$ extracted by Shanks in [7] were checked against the table.

3. Distribution. The distribution of the class numbers of the fields is given in Table I. For squarefree integers $d$ in a given interval, let $N_{g}$ be the number of fields $Q(\sqrt{ } d)$ with $g$ genera, and let $N_{f g}$ be the number of these which have $f$ ideal classes per genus. The entries in Table I are the values $N_{g}$ and $N_{f g}$ for values of $d$ in intervals of 10000 integers. If we consider the proportions $N_{f g} / N_{g}$ we find for small values of $h$, these proportions appear to be independent of $d$, although in the tail of the distribution with $h$ relatively large, the proportion increases with $d$. A plot of the value $N_{f g} / N_{g}$ against $f$ on log-log paper suggested that for small values of $h, N_{f g} \doteqdot N_{g} / f^{2}$. This would mean that for $g=1, N_{f g} / N_{g} \doteqdot 8 \pi^{-2} f^{-2}$ (odd values of $f$ only), and for $g>1$, $N_{f g} / N_{g} \doteqdot 6 \pi^{-2} f^{-2}$.

In Table II we compare the proportions $N_{1 f} / N_{1}$ for the total population, with

\begin{tabular}{crrrrrrrrrrrr} 
& \multicolumn{10}{c}{ TABLE II } \\
$\mathrm{f}=$ & 1 & 3 & 5 & 7 & 9 & 11 & 13 & 15 & 17 & 19 & + \\
$8 \pi^{-2} \mathrm{f}^{-2}$ & 81.1 & 9.0 & 3.2 & 1.7 & 1.0 & 0.67 & 0.48 & 0.36 & 0.28 & 0.22 & 2.0 \\
$\mathrm{~N}_{1 \mathrm{f}} / \mathrm{N}_{1}$ & 80.5 & 10.3 & 3.7 & 1.8 & 1.2 & 0.65 & 0.48 & 0.38 & 0.20 & 0.16 & 0.60 \\
$\mathrm{~N}_{\mathrm{f}}^{(1)} / 5000$ & 79.7 & 10.4 & 3.6 & 2.0 & 1.3 & 0.58 & 0.56 & 0.40 & 0.22 & 0.22 & 0.90 \\
$\mathrm{~N}_{\mathrm{f}}^{(2)} / 5000$ & 79.9 & 10.5 & 4.0 & 1.7 & 1.1 & 0.56 & 0.60 & 0.42 & 0.38 & 0.12 & 0.76
\end{tabular}


the values of $6 \pi^{-2} f^{-2}$. For comparison we also include $N_{f}^{(1)} / 5000$, and $N_{f}^{(2)} / 5000$, where the $N_{f}^{(1)}$ are the corresponding values obtained from Kloss's table [7] and the $N_{f}^{(2)}$ from Lakein's table [6]. The three distributions agree closely although they are from dissimilar populations. Each value is expressed as a percentage.

In Table III we compare the proportions $N_{g f} / N_{f}$ for $g=2,4,8$ for the total population, with the values of $6 \pi^{-2} f^{-2}$ each proportion being given as a percentage.

T ABLE III

$\begin{array}{cccccccccccc}\mathrm{f}= & 1 & 2 & 3 & 4 & 5 & 6 & 7 & 8 & 9 & 10 & + \\ \mathrm{g}=2 & 66.5 & 13.0 & 8.4 & 3.2 & 3.0 & 1.7 & 1.3 & 0.75 & 0.86 & 0.47 & 1.7 \\ 4 & 64.6 & 15.0 & 8.3 & 4.5 & 2.8 & 1.8 & 1.1 & 0.60 & 0.55 & 0.23 & 0.57 \\ 8 & 67.1 & 18.2 & 8.4 & 3.4 & 1.8 & 0.60 & 0.18 & 0.18 & 0.05 & 0.00 & 0.09 \\ 6 \mathrm{f}^{-2} \pi^{-2} & 60.8 & 15.2 & 6.8 & 3.8 & 2.4 & 1.7 & 1.2 & 0.95 & 0.75 & 0.61 & 5.8\end{array}$

It can be seen that when $h$ is small $(h<25), N_{f g f} / N_{g}$ is independent of $g$ for $g=2,4$ and 8 . Again, the behavior of the tail, for large values of $h$ differs markedly from $6 f^{-2} \pi^{-2}$, indeed if $N_{g f}=6 f^{-2} \pi^{-2} N_{g}$, we would find the average value of $f$ over all fields in the interval with $g$ genera was

$$
\left(\sum_{f=1}^{\infty} f \cdot N_{g f}\right) / N_{g}=6(8) \pi^{-2} \sum_{f=1}^{\infty} f^{-1}=\infty,
$$

as opposed to the measured average values, 1.96, 2.09, 1.89, 1.59 and 1.31 for the values $g=1,2,4,8$ and 16 .

If we compute the values of $\pi^{-1} f^{-1}\left(6(8) N_{g} / N_{f g}\right)^{1 / 2}$ for $5<e<10$, we find that for values of $h \leqslant 25$, this value ranges from $1.46\left(N_{1,23}=6\right)$ to $0.87\left(N_{8,3}=118\right)$, with an average value of 0.94 . Hence, we conjecture, for a range of values of $d$ in the neighborhood of the integer $a$,

\begin{tabular}{|c|c|c|c|c|c|c|c|c|c|}
\hline \multicolumn{10}{|c|}{ TABLE IV } \\
\hline $\mathrm{f}$ & $d$ & $\mathrm{f}$ & $d$ & $f$ & d & $f$ & $d$ & $f$ & $d$ \\
\hline$g=1$ & & 43 & 14401 & 13 & 13321 & 36 & 45511 & 19 & 99295 \\
\hline 1 & $2 *$ & 45 & 32401 & 14 & 11794 & 37 & $38026 *$ & 20 & 94546 \\
\hline 3 & $79 *$ & 47 & 78401 & 15 & 11321 & 38 & 93619 & 21 & 77779 \\
\hline 5 & 401 & 49 & 70969 & 16 & 25282 & 47 & 99226 & 24 & $50626^{*}$ \\
\hline 7 & 577 & 51 & 69697 & 17 & 19882 & $g=4$ & & $g=8$ & \\
\hline 9 & 1129 & 53 & 69694 & 18 & 25279 & $\frac{8}{1}$ & 130 & $\frac{5}{1}$ & 1155 \\
\hline 11 & 1297 & 57 & 41617 & 19 & 19834 & 2 & 399 & 2 & 4354 \\
\hline 13 & 4759 & 63 & 57601 & 20 & 41599 & 3 & $730 \%$ & 3 & 10455 \\
\hline 15 & 9871 & 87 & 90001 & 21 & 47959 & 4 & 3026 & 4 & 16555 \\
\hline 17 & 7054 & $g=2$ & & 22 & 59203 & 5 & 3970 & 5 & 19210 \\
\hline 19 & 15409 & 1 & $10 *$ & 23 & 49321 & 6 & 9790 & 6 & 33490 \\
\hline 21 & 7057 & 2 & $82 *$ & 24 & 79522 & 7 & $5626 *$ & 7 & 48399 \\
\hline 23 & 23593 & 3 & 235 & 25 & 54769 & 8 & 16555 & 8 & 81130 \\
\hline 25 & 24859 & 4 & $226^{*}$ & 26 & 77842 & 9 & 18226 & 9 & 65026 \\
\hline 27 & $8761 *$ & 5 & 1111 & 27 & 49834 & 10 & 16899 & 11 & 56170 \\
\hline 29 & 49281 & 6 & 1522 & 28 & 84679 & 11 & $11026 *$ & $g=16$ & \\
\hline 31 & 97753 & 7 & $1534 \%$ & 29 & 27226 & 12 & $21610 *$ & 1 & 15015 \\
\hline 33 & 55339 & 8 & $2305^{*}$ & 30 & 78745 & 13 & $23410 *$ & 2 & 39270 \\
\hline 35 & 25601 & 9 & 4954 & 31 & 68179 & 14 & 39999 & 3 & 81510 \\
\hline 37 & 24337 & 10 & $3601 \%$ & 33 & 87271 & 15 & 88231 & & \\
\hline 39 & 41614 & 11 & $4762 *$ & 34 & 53362 & 16 & 71290 & & \\
\hline 41 & 55966 & 12 & 9634 & 35 & 56011 & 17 & 63505 & & \\
\hline
\end{tabular}


Conjecture A. For $a$ large, $h$ small, $N_{g f} / N_{g}$ is independent of $a$.

Conjecture B. For $a$ large, $h$ small, $N_{g f} \doteqdot N_{g 1} / f^{2}$.

4. Extreme Values. In Table IV we list the least determinant $d(d<100000)$ which generates the quadratic number field $Q(\sqrt{ } d)$ with $g$ genera and $f$ ideal classes per genus.

In Table IV the values of $d$ with an asterisk are those values $d$ for which $h(d)$ is larger than any preceding class number.

$h$ can be obtained analytically from the Dirichlet series $L(1, \chi)$ where $\chi$ is the character of the field $Q(\sqrt{ } d)$ using the formula, $h=L(1, \chi) \sqrt{ } D / 2 \ln \epsilon, D$ being the discriminant of $Q(\sqrt{ } d), \epsilon$ the fundamental unit. In [7] Shanks notes $L(1, \chi)=$ $O(\ln \sqrt{ } d)$. Also as $\epsilon=(x+y \sqrt{ } D) / 2$, with $x, y \geqslant 1, x^{2}-D y^{2}= \pm 4 \Rightarrow x>\sqrt{ } D-2$, $\epsilon>\sqrt{ } D-1$, and hence $h=O(\sqrt{ } d)$. In Table $\mathrm{V}$ we give those values of Table IV for which $h / \sqrt{ } d>0.4$.

\begin{tabular}{|c|c|c|c|c|c|c|c|c|}
\hline \multicolumn{9}{|c|}{ TABLE V } \\
\hline d & 2 & 10 & 226 & 82 & 730 & 50626 & 11026 & 399 \\
\hline $\mathrm{h}$ & 1 & 2 & 8 & 4 & 12 & 96 & 44 & 8 \\
\hline $1 / \sqrt{d}$ & 0.71 & 0.63 & 0.53 & 0.44 & 0.44 & 0.43 & 0.42 & 0.40. \\
\hline
\end{tabular}

Mathematics Department

Massey University

Palmerston North, New Zealand

1. C. F. GAUSS, Disquisitiones Arithmeticae, Fleischer, Leipzig, 1870; English transl., Yale Univ. Press, New Haven, Conn., 1966. MR 33 \#5545.

2. M. D. HENDY, "Applications of a continued fraction algorithm to some class number problems," Math. Comp., v. 28, 1974, pp. 267-277. MR 48 \#8440.

3. E. L. INCE, Cycles of Reduced Ideals in Quadratic Fields, Mathematical Tables, vol. IV, British Association for the Advancement of Science, London, 1934.

4. K. E. KLOSS, "Some number-theoretic calculations," J. Res. Nat. Bur. Standards Sect. B, v. 69B, 1965, pp. 335-336. MR 32 \#7473.

5. K. E. KLOSS, M. NEWMAN \& E. ORDMAN, Class Number of Primes of the Form $4 n+1$, National Bureau of Standards, 1965. (Deposited in the UMT file.)

6. R. B. LAKEIN, "Computation of the ideal class group of certain complex quartic fields," Math. Comp., v. 28, 1974, pp. 839-846.

7. D. SHANKS, Review of UMT file [5], Math. Comp., v. 23, 1969, pp. 213-214. 